\title{
Le traitement de la variation linguistique dans les travaux universitaires sur les langues pratiquées en Algérie : Cas de quelques positions de recherche
}

\author{
Chachou Ibtissem, Bensekat Malika \\ Université de Mostaganem, chercheures-associée au CRACS, Laboratoire DFP \\ ibtissemchachou@yahoo.fr, bensekat malika@yahoo.fr
}

\begin{abstract}
Résumé. Notre contribution porte sur les dynamiques de recherche sur la question de la variation (ou leur absence) par rapport aux langues pratiquées en Algérie. Ces langues sont l'arabe algérien, le français, les langues berbères et l'arabe institutionnel, et ce dans les domaines formels et informels. Notre corpus renferme un échantillon représentatif des écrits portant sur des pratiques plurilingues et/ ou les théorisant, lesquelles pratiques sont saisies et analysées des points de vue sociolinguistique et sociodidactique. Ces écrits/résultats sont l'œuvre de chercheurs algériens dont les travaux sont repris par les jeunes chercheurs notamment et qui leur servent souvent de références et d'appuis théoriques. Nous tenterons à travers cette contribution, autant que faire se peut, de dégager ces positionnements théoriques, d'en interroger les soubassements d'ordre épistémique et d'identifier clairement les orientations, les priorités et les attentes pour la recherche en didactique et en sociolinguistique. Il s'agira notamment de mesurer leur apport pour la recherche dans le domaine des sciences du langage, leur pertinence ainsi que leur utilité, mais d'observer également ce qui peut handicaper la réflexion sur des questions aussi sensibles tant elles sont innervées par des considérations idéologiques dont le linguiste n'échappe pas toujours.
\end{abstract}

\begin{abstract}
Our contribution focuses on the research dynamic on the issue of the change (or lack thereof) with respect to the languages spoken in Algeria. These languages are Algerian Arabic, French, Berber languages and institutional Arabic, and in formal and informal areas. Our body contains a representative sample of the literature on multilingual practices and / or the theorizing, which practices are entered and analyzed the points of sociolinguistics and sociodidactique view. These writings / results are the work of Algerian researchers whose work is taken up by young researchers in particular, which often serve as their reference and theoretical support. We will try through this contribution, whenever possible, to identify these theoretical positions, to query the order epistemic foundations and clearly identify directions, priorities and expectations for education research and sociolinguistics. This will include measuring their contribution to research in the sciences of language, relevance and usefulness, but also observe what may handicap thinking on issues as sensitive as they are innervated by considerations whose ideological linguist do not always escape.
\end{abstract}

\section{Introduction :}

Dans un article livré à la revue «Réflexions et perspectives», parue en 2012 à l'occasion du cinquantenaire de l'Algérie indépendante, Dalila Morsly a dressé un état des lieux de la recherche sociolinguistique où elle a passé en revue "les axes, les thématiques, problématiques et méthodologies » (Morsly 2012 : 246) qui caractérisent «une sociolinguistique algérienne» (2012:251) menée par des chercheurs qu'elle dit "en résonance avec leur société et avec les débats que suscitent les langues» (2012:258) et qui «sont réactifs par rapport aux décisions institutionnelles qui concernent la définition des statuts et rôles des langues» (Idem). Un constat qu'elle établi en conclusion à une série d'investigations menées sur le terrain et d'observation de nombreux travaux réalisés dans les universités algériennes et ailleurs. Cette réflexion sera poursuivie dans le cadre du colloque international : Algérie: 50 ans de pratiques plurilingues. Son objectif est de continuer «de rassembler les résultats et les conclusions auxquels ont abouti ces recherches pour essayer de repérer les tendances et dynamiques 
linguistiques dans les différents champs de la communication sociale ${ }^{1}$. Dans le cadre de cette contribution, nous reprenons une des questions que Dalila Morsly a longtemps analysé dans ses travaux à savoir la variation ${ }^{2}$. Nous la soumettrons à un essai d'évaluation en en examinant le traitement qui en est fait dans le discours universitaire produit sur les langues pratiquées en Algérie.

Notre objectif est de nourrir cette réflexion en répondant aux questions suivantes : Quels dispositifs théoriques et méthodologiques sont mis en œuvre dans les recherches en cours, en sociolinguistique algérienne et en sociodidactique, pour décrire et analyser la distribution des langues/variétés ainsi que les phénomènes résultant de leur contact? Quels sont leurs apports et quelles seraient leurs limites pour la recherche universitaire? Il s'agit de comprendre les dynamiques de recherche sur la question de la variation (ou son absence). Les langues concernées sont: l'arabe algérien, l'arabe institutionnel, les mixtes linguistiques, le français et les langues berbères. Nous avons considéré le discours universitaire comme un texte scientifique "produit dans le cadre de l'activité de recherche à des fins de construction et de diffusion du savoir » (Boch \& Rinck 2010 : 5). Les deux types de variation qui y sont saisis sont relatifs à la variation diachronique, liée au temps, et à la variation diatopique, relative aux différenciations géographiques et régionales (Calvet 2013 :77). Leur analyse se trouve également intégrée à l'analyse de la variation sociale à laquelle s'intéresse la sociolinguistique en milieu ordinaire et récemment la sociodidactique (Rispail 2012), en contextes scolaire et universitaire.

La thématique de la variation linguistique est considérée par Dalila Morsly comme étant «importante » (Morsly, 2013) car à l'origine du «fondement de la discipline » (Idem). Cependant, sous couvert d'argumentation a priori «scientifique », se donnent à lire, tantôt, des positions idéologiques qui sont parfois revendiquées en tant que telles et tantôt, des analyses stéréotypées. Cela peut s'expliquer par le fait que ces analyses soient dépassées par la dynamique du terrain et de l'évolution des usages, ou bien qu'elles soient empruntées à d'autres contextes et calquées sur le réel algérien sans être repensées (Chachou 2013), et ce pour différentes raisons ${ }^{3}$. Nous nous appuierons sur les critiques formulées par Dourari Abderrezak (2011), Morsly Dalila (2013) et Djamel Kouloughli (2009), pour mettre à jour d'autres facteurs susceptibles de se révéler à l'examen du discours académique, notamment les relents idéologiques et subjectifs qui apparaissent dans le discours et qui stéréotypent la réflexion sur le sujet. Nous tenterons, autant que faire se peut, de dégager ces positionnements théoriques, d'en interroger les soubassements d'ordre épistémique et d'identifier clairement les orientations, et partant, les priorités et peut-être les insuffisances pour la recherche en didactique et en sociolinguistique. La priorité concernant l'arabe algérien et les langues berbères serait de «sortir du modèle des miroirs dominants » ${ }^{4}$ (Di Meglio 2011 ) et de «soustraire les questions linguistiques à la querelle politicienne» tout en atténuant «l'intervention du militantisme dans la réflexion linguistique » (Dourari 2011). Aborder cette question participerait également à la déconstruction des récits identitaires modernes dont la langue est l'élément le plus emblématique (Temlali 2015).

\footnotetext{
${ }^{1}$ Voir l'argumentaire du colloque : http://www.sladdlab.net/

${ }^{2}$ En conclusion à un article consacré à la question de la didactique de la variation des langues berbères, Dalila Morsly avance : «Le débat (...) illustre avec force que la variation linguistique, y compris dans un contexte sociolinguistique apparemment favorable à sa prise en compte, constitue un point de résistance puissant » (Morsly, 2011).

${ }^{3}$ Même si les chercheurs algériens travaillent dans leur grande majorité sur des corpus algériens, souvent le cadre théorique est emprunté à la tradition française ou anglo-saxonne. Une tradition critique et réflexive (articles, études) sur les cadrages théoriques reste marginale. Voir à ce propos : (Dourari 2011), (Morsly 2013), (Chachou : 2013).

${ }^{4}$ L'évolution des études sur la langue corse en contexte universitaire me semble très intéressante à observer. Le caractère idéologique tend à s'estomper au profit d'une réflexion scientifique sur la question. Elle se traduit notamment par des expériences dans le cadre de projet d'éducation (bi)plurilingue : "Par l'efficacité d'un appareil théorique forgé à partir de diverses sources et en faveur de situations minorées, par le relais des enseignements et des formations, par l'évolution du statut de la langue, nous avançons que les effets de l'idéologie diglossique (Thiers, 1989 : 246) tendent à être dépassés pour une approche plus ouverte de la LCC» (LCC=La langue et la culture corses). Voir : Di Meglio, A, «Langue(s), identité(s) et scolarité : la question du rapport à l'Autre et de la citoyenneté à partir de l'exemple corse ", in : Communication in L'École : instrument de sauvetage des langues menacées ? Colloque - 30/09-1/10 2005, Université de Perpignan : http://halshs.archives-ouvertes.fr/halshs-00646543
} 


\section{Présentation du corpus, choix et justification :}

Notre corpus se limite à quelques linguistes algériens. Dalila Morsly, Yasmina Cherrad, Khaoula Taleb Ibrahimi, Dourari Abderrezak, Djamel Khoulougli, Mohamed Miliani, Kara Attika-Yasmine, Salem Chaker et Abdou Elimam. A l'exception de Salem Chaker qui s'est exclusivement intéressé au berbère, les autres linguistes ont abordé les différentes langues en usage en Algérie. Le choix de ces auteurs est dicté par des contraintes liées à l'espace restreint de cet article et qui ne nous permet pas de passer en revue toute les positions de recherche sur la question. Leurs travaux présentent des aspects théoriques, épistémiques et critiques qui nous semblent importants à reproduire et d'autres intéressants à interroger. Rappelons le contexte sociologique qui a présidé à l'émergence de la sociolinguistique aux Etats-Unis et en Europe (Baylon 1996). Des projets de recherche motivés par des visées interventionnistes et utiles ont été à l'origine des travaux pionniers dans le domaine : Quelle utilité pour ces sciences aujourd'hui en Algérie ? Quelle pertinence pour la recherche ? Et quelle originalité dans la saisie de ces phénomènes ?

Nous tenterons dans le cadre restreint imparti à cette contribution de nous intéresser à quelques aspects de la question de la variation dans les différentes langues en contact en Algérie. Nous nous intéresserons respectivement aux travaux qui ont porté sur le français, l'arabe algérien et les langues berbères.

\subsection{Des débuts des interrogations sur le français en Algérie}

L'Algérie entretient avec ses langues, et avec le français en particulier, des rapports complexes et toujours en évolution. En effet, depuis l'indépendance de l'Algérie en 1962 et après une présence de 132 ans, le français, langue d'ex colonisateur, possède un statut privilégié par rapport aux langues étrangères en présence. Il faut dire que la langue française a profondément marqué l'inconscient de plusieurs générations d'Algériens en raison de la domination coloniale et des diverses politiques linguistiques et culturelles mises en place dès les premières années de l'occupation.

Placé, depuis 1962, dans un rapport conflictuel avec la langue arabe (langue nationale et officielle), le français est désormais défini sur le plan institutionnel comme une langue étrangère. Toutefois, ce statut reste théorique dans la mesure où la situation linguistique se caractérise par une forte prééminence de l'usage de la langue française dans le pays, avec des variations suivant les zones géographiques (Temim, 1998). L'étendue et la diversité des champs d'action de cette langue ainsi que son prestige semblent être les facteurs dynamisants et déterminants qui lui confèrent une position (informelle) dans la hiérarchie des valeurs et sur le marché linguistique algérien.

Les études élaborées posent d'ailleurs l'hypothèse d'un français local en construction depuis l'indépendance. On postule "l'existence d'un français parlé et écrit distinct du français national » (Morsly 1988). Ce qui sous-tend que le français fait l'objet d'une appropriation qui se traduit par l'apparition de particularités linguistiques, ce sont ces particularités que les descriptions s'efforcent de mettre en évidence, empruntant pour cela une démarche qui consiste, le plus souvent, à repérer les écarts par rapport aux normes d'un français défini comme «national », «central» ou «standard ».

Dans la masse de ces travaux qui s'intéressent de façon plus ou moins ponctuelle ou générale aux différents niveaux de l'analyse linguistique, on retrouve dans ce qui est signalé comme des spécificités, des constantes qui, au-delà de la variation propre à chaque région, peuvent donner une idée des éléments linguistiques communs susceptibles d'aider à identifier et à poser l'existence d'un «français algérien ». En effet, la dynamique sociolinguistique du français en Algérie témoigne de «l'ancrage de cette langue parmi les emplois effectifs voire créatifs qui s'y développent au quotidien. Ils oscillent entre éclatement et métissage et transitent par l'intervalle des variations, elles mêmes intrinsèques au changement» (Chachou 2011). 
Ce mixage linguistique recouvrant de nombreux phénomènes issus des contacts des langues en présence est le sujet qui prête le plus à ambigüité : «sabir franco-arabe » (Taleb Ibrahimi 1997: 115), «francarabe » (Moatassim 1992 : 134), « semilinguisme » (Concept emprunté à El-Hedi Saada par Taleb Ibrahimi 1997: 167), «demilinguisme », «analphabétisme bi(tri)lingue », "la mixoglossie », « schizoglossie » (Miliani 2002 : 81, 94, 95), «multilinguisme éclaté » (Moatassim 1992 : 134), etc. Il importe de noter ici que

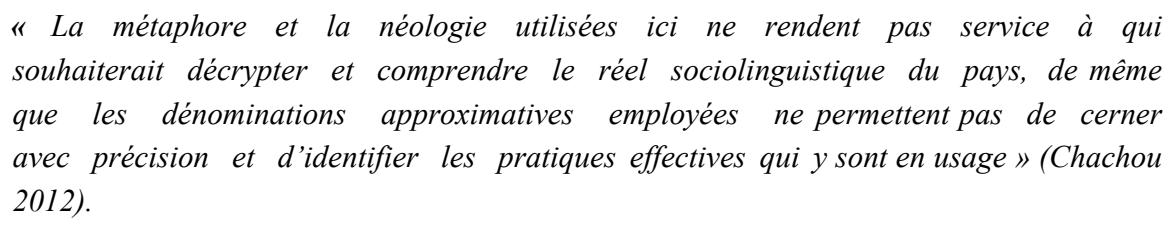

Selon Abderrezak Dourari :

"Ce sont [...] l'analyse et la conceptualisation des pratiques langagières
effectives des Algériens qui constitueront les principes devant guider la
reconstruction du champ de la pensée sociolinguistique algérienne et non pas les
concepts issus de description de situations particulières empruntées à d'autres
sociétés". (Dourari 2002).

Les éclairages qu'apportent les travaux de Dourari Abderrezak, portant sur les pratiques réelles des locuteurs plurilingues en Algérie, et qui rejoignent les réflexions menées et les constats établis par Dalila Morsly, Khaoula Taleb Ibrahimi et Yasmina Cherrad-Benchefra, étaient l'occasion de montrer que s'aventurer hors de la classe conduit à rencontrer des formes langagières et conversationnelles non prévues dans le cadre scolaire et qui sont susceptibles d'heurter les normes habituellement admises et donc diffusées - voire imposées. Ces chercheurs ont été amenés à décrire en partie les pratiques sociales langagières algériennes : toute la question sera de savoir quelle autonomie leur est accordée par rapport à la norme de l'école oùes pratiques linguistiques sont associées à un « français de France »?

Ces travaux ont souligné à juste titre que l'accent est implicitement mis par l'institution sur le «linguistiquement correct» (Dourari 2002 : 20) et le «linguistiquement dicible» (Idem). Cet intérêt porté exclusivement aux structures de la langue permet de «garder impensées les questions qui mettent en crise le discours déréalisé du pouvoir (et d'une certaine opposition) et de centrer l'intérêt sur la combinatoire linguistique » (Idem). C'est dans ce contexte intellectuel que l'on mesure toute la pertinence du point de vue de Khaoula Taleb Ibrahimi quand elle affirme :

\footnotetext{
" La notion de pratique langagière marque une évolution dans la description linguistique et sociolinguistique car il ne s'agit plus uniquement d'analyser les règles internes au système linguistique qui organisent la compétence d'un locuteur idéal (...) ou de décrire les régularités structurales d'un corpus fermé de données (...), mais de s'intéresser à la diversité des locuteurs, à la diversité de leurs conduites». Elle ajoute : "L'étude de pratiques langagières permet de rassembler une somme d'informations et de renseignements sur la réalité sociolinguistique d'une société donnée, en ce sens elles font partie d'un ensemble plus important qui englobe toutes les pratiques humaines» (Taleb Ibrahimi 1995 : 120).
} 
Dans la présentation du numéro 17-18 de la revue Insaniyat (Taleb Ibrahimi 2002) consacrée à l'analyse des pratiques langagières des locuteurs algériens et maghrébins, Khaoula Taleb-Ibrahimi insiste sur la nécessité de dépasser l'analyse idéologique au profit de la description des vraies pratiques ${ }^{5}$, même si, nous le voyons pour les autres langues telles que l'arabe algérien et les langues berbères, l'évaluation du poids de l'idéologie n'est point superfétatoire. Aussi, dans le souci de brosser un tableau de la configuration sociolangagière de la société à partir de situations de communication concrètes et variées, elle résume cette notion de variation linguistique ou de pratiques langagières effectives autour des questions suivantes :

\begin{abstract}
"Que font-ils quand ils parlent? Quand ils écrivent? Quand ils chantent? Comment se structurent, à travers leurs conduites langagières, leurs rapports à leur(s)langue(s)ainsi qu'aux normes linguistiques dominantes dans nos sociétés? Comment gèrent-ils ou ne gèrent-ils pas leur multilinguisme? Quelles sont les stratégies de communication qu'ils mettent en æuvre? Comment se structurent-elles? Quelle est la place qu'occupent les phénomènes de contact de langues dans leurs pratiques et leurs stratégies? Y-a-t-il des indicateurs des stratifications sociales dans les comportements langagiers des locuteurs algériens? ».
\end{abstract}

Elle souligne en outre que «Les locuteurs algériens ont en commun la possibilité d'user d'une gamme de variétés qui constituent leur répertoire verbal ». Khaoula Taleb Ibrahimi met en évidence la capacité des locuteurs/scripteurs à se mouvoir dans leur répertoire verbal en en exploitant toutes les facettes ainsi que leur liberté souvent marquée d'impertinence à l'égard des normes imposées. Ces pratiques semblent de facto dénoter le déplacement vers une nouvelle acception de la « Norme » qui ne serait plus imposée mais qui s'apparenterait à une «norme de compréhension, de possibilité de transfert entre les langues, norme d'échange, norme-création, norme-interaction, norme-ouverture, norme-mobilité, norme plurielle, riche de la diversité de ce pays ». (Taleb Ibrahimi 2002).

Concernant le contexte d'enseignement/apprentissage, on observe que cette problématique pose la question du rapport entre la norme et le français à enseigner en Algérie.

Cette question de la norme a interpellé les spécialistes qui se sont intéressés particulièrement à l'aspect « normatif » lié à toutes les facettes de l'enseignement. Le poids des normes - au sens le plus large : linguistique, culturelle - est-il si important dans un cours de langue ? Quelles sont ses conséquences dans la réalisation des cours mais aussi dans les manuels de langue, dans l'appareil pédagogique de l'enseignant ? Quelle place donner à la variation ? Quel français enseigner ? La question n'est pas si anodine que cela. Elle invite l'enseignant à réfléchir à la base même de son enseignement dans un monde plus global que jamais.

Ces réflexions ont montré qu'une gestion linguistique soumise à la norme et un intérêt porté aux structures et à la combinatoire de la langue ne permet guère de prendre en considération les liens entre la

\footnotetext{
${ }^{5}$ « Nous voulions, avec ce numéro, sortir des sentiers battus de l'analyse sociopolitique et idéologique de la question pour aborder plus spécifiquement la manière avec laquelle se structurent les relations entre les faits langagiers et les faits sociaux (la société dans son ensemble) » (Taleb Ibrahimi 2002).
} 
(les) langue(s) et la société. C'est ce que souligne Attika-Yasmine Kara par rapport au contexte d'enseignement algérien:

\begin{abstract}
"Dans la politique linguistique algérienne et dans l'enseignement, ni le statut des langues en présence, ni la variation sociolinguistique et donc les pratiques langagières réelles des apprenants, ne sont prises en compte. C'est la norme qui est largement ancrée dans la tradition de l'enseignement algérien que ce soit pour l'enseignement de l'arabe ou des autres langues étrangères, ce qui complexifie l'enseignement/apprentissage de ces langues, provoque des problèmes à tous les niveaux de l'éducation et des déperditions non sans canoséquences sur l'acquisition du savoir plusridisplinaire". (Kara $2010: 85$ ).
\end{abstract}

Dans le cadre de certains de ses travaux, Attika-Yasmine Kara a mené une réflexion autour des enjeux de la variation sociolinguistique comme pratique d'enseignement/apprentissage des langues. Elle montre que la prise en compte de la variation sociolinguistique est une donnée incontournable pour une «parfaite intégration professionnelle des diplômés » (Kara 2010), ceci dans le mesure où les productions linguistiques plurilingues sont présentes dans le contexte algérien, aussi bien en milieu social qu'en milieu d'apprentissage. Dans ce cadre, c'est le centrage sur l'apprenant qui fait reconnaître le fait que l'apprentissage est la constitution d'un réservoir langagier composite dans lequel toutes les capacités linguistiques trouvent ou trouveront leur place.

Au-delà de cette question de norme, on a postulé l'existence d'un «français parlé d'Algérie » (Queffélec et al. 2002) (Bensekat 2012) ou d'un «français parlé en Algérie » (Morsly 1983) (Cherrad-Benchefra 1992). Le premier étant «encore non reconnu et sans statut, mais qui serait à décrire et affirmer, dans une optique variationniste qui dépasse le seul cadre algérien» (Bensekat 2012: 142). A ce propos, Yasmina Cherrad note que «la rencontre entre les langues et le français produit une parole émaillée de toutes sortes de modifications à divers niveaux: (phonologique, syntaxique, sémantique), cette création repose globalement sur les potentialités offertes par les systèmes linguistiques en présence». Il s'agit tantôt du français académique qui jouit d'une certaine co-officialité aux côtés de l'arabe institutionnel, et tantôt du français parlé d'Algérie. Elle souligne que

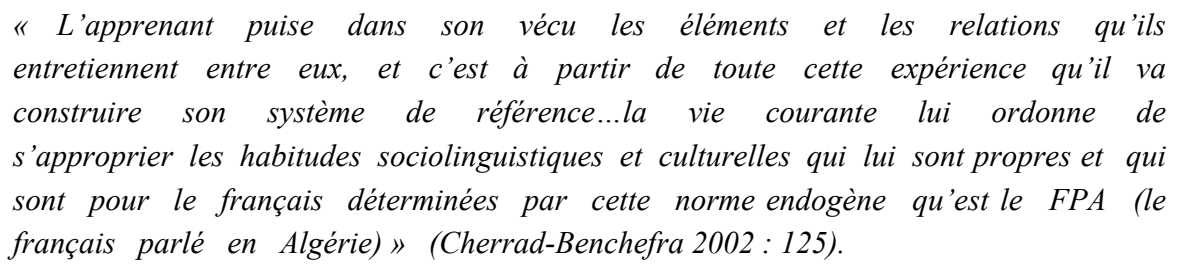

Face à cette situation, Mohamed Miliani propose deux scénarii de gestion aux décideurs : Le premier consisterait en une "prise en charge réelle par un aménagement linguistique harmonieux qui se baserait sur un bilinguisme actif pour ne pas avoir à subir les affres d'une unilingualisation déstructurante » (Miliani 2002: 94). Le second résiderait dans le fait "qu'en tant que communauté linguistique particulière, les Algériens développent l'autre norme qu'ils sont en train de créer et d'enrichir de manière progressive et quotidienne » (Idem). 
En effet, dans la continuité des travaux sur la didactique des langues et le plurilinguisme en Algérie, la recherche scientifique universitaire devrait proposer une réflexion sur l'enseignement/apprentissage des langues, dans une perspective sociodidactique et une optique variationniste, qui met au premier plan les liens entre l'espace de la classe et les autres espaces sociaux. Ceci l'amènerait à s'interroger sur les relations entre la situation sociolinguistique plurilingue de l'Algérie et les modalités effectives d'enseignement/apprentissage du français. Il est important également d'apporter quelques pistes de réponse à la question de savoir par quels types de rapport sont reliés le plurilinguisme et les situations où il se développe, ainsi que la nature des rapports entre l'enseignement des langues et les pratiques langagières de leurs usagers, de savoir vers quel type de socio-didactique des langues et des usages langagiers en situation plurilingue ces rapports peuvent-ils nous guider ? Il s'agit de montrer comment les répertoires plurilingues des apprenants (arabe algérien, langues berbères, français algérien) peuvent à la fois jouer favorablement sur les apprentissages et constituer une reconnaissance, dans l'institution scolaire, de la variété « algérienne » du français.

Ces propositions/interrogations ne sont pas seulement didactiques, car elles ouvrent un horizon de possibles : une école qui ne serait plus l'espace d'application des politiques linguistiques, mais son inspiration ; l'école comme médiation entre les pratiques linguistiques réelles et les instances de pouvoir, espace de recréation des savoirs plus que d'exécution de consignes. Un nouveau contexte pour l'enseignement se dessine alors, dû à une mutation des représentations qu'il véhicule, à des définitions notionnelles qui «bougent », entraînant une approche variationniste des langues et une prise en compte l’hétérogénéité des ressources langagières des apprenants en classe.

\subsection{Concernant l'arabe algérien :}

La question de la dénomination des langues implique elle-même la prise en compte ou non de la variation, c'est-à-dire l'apparition et la reconnaissance de nouvelles variétés résultant de l'identification de la variation (Blanchet 2004) en tant que langue ou son inclusion en tant que variété dans un ensemble, un phénomène que l'on nomme: "satellisation diglossique " (Marcellesi 2003) ou "polarisation diglossique » (Boyer 2008 : 53). Cette phase de dénomination conduit à la classification/ catégorisation de «la langue» dans le discours scientifique soit en tant que variété autonome envisagée dans un continuum d'usages, ou bien, en tant que variété dépendante d'autres variétés et que l'on intégrerait de fait dans un continuum linguistique, ce qui implique sur d'autres plans soit sa prise en charge institutionnelle, statutaire ou bien son déni en tant que langue devant/ pouvant être prise en charge (Elimam 2004). L'orientation des recherches dans le domaine, leur diversité en dépendra aussi. L'arabe algérien sera considéré soit comme un objet d'étude susceptible d'être interrogé, soit, il sera traité comme une composante linguistique, simple réel à décrire au passage lorsqu'on évoque la situation sociolinguistique de l'Algérie.

Le discours scientifique en tant que constructeur et diffuseur du savoir ${ }^{6}$, en tant que force de propositions peut s'avérer, lorsqu'il est plombé par l'idéologie, stérile et stérilisant pour l'action en société sur les langues. Il handicaperait la réflexion à des perspectives d'institutionnalisation dans le cadre d'une proposition de politique linguistique par exemple. L'arabe algérien, parent pauvre de la revendication linguistique en Algérie, pâtitégalement d'un traitement mitigé sinon minorant dans le domaine de la recherche universitaire algérien. Considéré comme un dialecte de l'arabe, il a traditionnellement été inscrit dans un continuum linguistique de type diglossique où il a été situé au bas du classement et décrit comme étant une norme inférieure. Dans de nombreux écrits, outre une dénomination stigmatisante (Chachou 2012), l'arabe algérien est considéré comme une expression de fortune adoptée par les Algériens en remplacement de l'arabe classique dont ils auraient été spoliés/dépossédés, c'est ce qui nous

\footnotetext{
${ }^{6}$ " A la différence d'autres types de discours, où les croyances et les savoirs communiqués ne sont pas nécessairement pris en charge comme vrais par le destinataire, on peut supposer que le propre de tout discours scientifique serait que ces croyances et savoirs sont partagés par le destinataire, c'est-à-dire, ici, pris en charge comme vrais par au moins une partie du groupe auquel le discours s'adresse ». (Gâță 2006)
} 
fait penser à une perception victimaire (Benrabah 1999) (Chachou 2013) et une approche assez expéditive dont il fait l'objet. Des travaux sur l'histoire de l'arabe algérien manquent ou ne sont pas cités quand ils sont réalisés. Nous pensons aux recherches sur l'enseignement de l'arabe institutionnel, de l'arabe algérien, le dit «arabe vulgaire » (Troupeau 1997) et le processus de théorisation diglossique qu'il a suscité durant la période coloniale (Girard 2013). Diverses approches peuvent être sollicitées pour explorer un corpus très riche en manuels, dictionnaires et en réflexions d'ordre linguistique et sociologique que l'on retrouve dans les pages de présentations de ces supports d'enseignement (Messaoudi \& Larzul 2013).

Pour revenir au continuum, repris dans les travaux sur la réalité algérienne et oùst reconduite l'approche diglossique, nous rappelons que la tendance à la classification et à la stratification a été très répandue depuis l'introduction, sous l'influence de la sociolinguistique variationniste, par El Hassan Shahir Ata (Koulouhgli 1996) du concept de continuum linguistique dans le domaine des études arabes. Mais que ce soit dans les approches en termes de diglossie, dans les approches stratifiées ou dans les approches en termes de continuum (Idem), les qualificatifs ont toujours été connotés : "dialectal des éclairés ${ }^{7}$ ", «dialectal des illettrés », «arabe parlé des cultivés », "Educated spoken arabic» (Idem). Dans les approches stratifiées la variation relevait davantage de la variation stylistique, c'est-à-dire une variation de registres mais oùla stratification relègue toujours au plus bas de l'échelle linguistique l'arabe vernaculaire. La polarisation diglossique est maintenue. Le concept de diglossie ne semble pas ici opératoire dans la mesure où il n'amène pas à définir des régularités et à identifier des variétés. Djamel Kouloughli insiste sur l'intérêt d'un tel objectif : "...la délimitation de ces diverses variétés d'arabe et leurs frontières est nécessaire non pas seulement pour des raisons méthodologiques et les exigences de la description linguistique, mais aussi du point de vue de la réalité sociolinguistique » (Kouloughli 1997), les implications sociales, éducatives sont rarement considérées sinon délibérément éludées. Pour l'Algérie, Khaoula Taleb Ibrahimi pose : «l'inopérance des schémas classiques » (Taleb Ibrahimi 1997) qu'elle considère comme un « héritage d'une vision puriste et rhétoriciste profondément ancrée dans la critique littéraire des anciens » (Idem : 45). Elle évoque "la nécessité d'une approche dynamique des variétés linguistiques ». (Idem : 45). En décrivant la « sphère arabophone », elle précise qu’elle

"...a tendance à se structurer dans un continuum de registres (variétés
langagières) qui s'échelonnent du registre le plus normé au moins normé. En
premier lieu vient l'arabe fusha (ou classique), puis l'arabe standard ou moderne,
véritable langue d'intercommunication entre tous les pays arabophones,
ensuite ce que nous appelons "le dialecte des cultivés (Idem) ou "l'arabe parlé
par les personnes scolarisées, enfin le registre dont l'acquisition et l'usage sont
les plus spontanés, ce que l'on nomme communément les dialectes ou parlers qui se
distribuent dans tous les pays en variantes locales et régionales " (Taleb Ibrahimi:
207-208).

Cette classification qui se voulait critique et moins rigide que le continuum diglossique s'apparente en réalité à un continuum de registres qui ne remet pas en question la satellisation diglossique en cause mais qui hiérarchise, en vertu d'une autre schématisation, du haut vers le bas, les langues en présence que sont l'arabe institutionnel et l'arabe algérien qui sont fragmentés en plusieurs variétés : "classique, standard, substandard, celui des scolarisés, l'arabe dialectal » (Idem). Mohamed Benrabah conteste cette idée de continuum en arguant qu'elle "remet en cause la frontière qui sépare l'arabe classique et l'arabe

\footnotetext{
${ }^{7}$ C'est nous qui soulignons, nous ferons de même pour tous les extraits des citations sur lesquelles il nous parait important d'attirer l'attention du lecteur.
} 
algérien », (Benrabah 1999 : 133) et qu'un continuum suppose une communauté de référence, laquelle n'a pas eu d'existence au Maghreb. Même s'il plaide actuellement en faveur d'un rapprochement de l'arabe de l'école de l'arabe maternel (Benrabah 2009) et non la reconnaissance de l'arabe algérien comme langue à part entière ainsi qu'il l'avait soutenu dans ses travaux antérieurs (Benrabah 1999).

Quant à Abdou Elimam, il aborde son individuation linguistique sur le plan diachronique (involontaire) :

" on peut considérer (...) que l'influence des formes linguistiques arabes -parmi tant d'autres- a fait évoluer le punique » (Elimam 2003 :47). "langue pleine et entière, le maghribi est (...) langue native et maternelle; depuis son individuation près de deux fois millénaires. Elle aura permis la floraison d'une littérature, attestée, millénaire (période couvrant le "adab ez-zajal), en Andalousie, jusqu'au melhoun contemporain) et un fond lexical immense mais non exploré. » (Idem).

Cette thèse a donné lieu à de nombreuses publications où des propositions en vue de l'institutionnalisation de l'arabe algérien sont proposées (Elimam 2004). Tout en adhérant à cette thèse du maghribi ${ }^{8}$ développée par Abdou Elimam, Dourari Abderrezak, lui, met l'accent sur la variation diatopique du dit « arabe dialectal », il récuse dans ce sens:

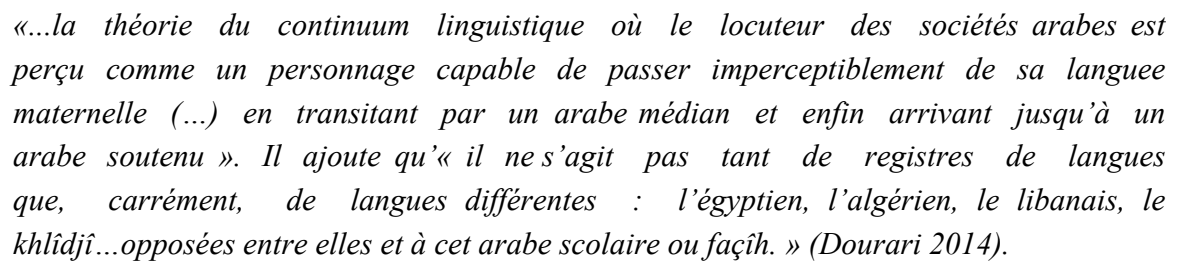

Il est important de signaler que dans le domaine de la recherche en sciences du langage, l'approfondissement critique des concepts est rarement entrepris comme il ressort des nombreux travaux de thèses et des articles scientifiques diffusés en Algérie 9 . En effet, la situation sociolinguistique, appelée assez facilement «paysage », est vite esquissée à la faveur d'un résumé fait des travaux de références les plus diffusés en Algérie et les plus disponibles aussi ${ }^{10}$. C'est ainsi que l'idée de continuum, la question de la dénomination, la complexité du plurilinguisme algérien et son appréhension sur le plan diachronique font rarement l'objet de discussions et de synthèses critiques et épistémiques. Ce qui n'est pas sans conséquences sur la nature des problématiques qui dominent le champ des sciences du langage en contexte algérien. La diversité des points de vue n'a pas été souvent ni encore moins systématiquement soumise à une réflexion critique, discutée. Ce que Pierre Bourdieu appelait: «Une sciences sociales de la

\footnotetext{
${ }^{8}$ La thèse selon laquelle la langue punique en usage au Maghreb est à l'origine les langues maghrébines qu'il appelle «maghribi » (Voir Elimam 2003).

${ }^{9}$ Se pose ici la question de la formation (avec tout ce que le terme implique) dispensée aux magistérants, mastérisants et aux doctorants algériens

${ }^{10}$ Outre les problèmes qui handicapent l'édition en Algérie de manière générale, il faut ajouter le fait que l'édition scientifique en matière des sciences humaines et sociales pâtit d'un manque de reconnaissance comparé au domaine des sciences dures. Voir : Boussad Wadi, L'édition algérienne : Etat des lieux, in : www.raina-dz.ne: «L'édition en Algérie a favorisé la publication de thèses académiques ou de livres parascolaires de piètre qualité, au détriment de collections de vulgarisation scientifique pour un large public. Les sciences sociales ont souvent été sacrifiées sur l'autel de la "technologie" et des sciences exactes ».
} 
linguistique » (Bourdieu 2002) aiderait à réfléchir au champ notionnel de la sociolinguistique algérienne où les concepts gagneraient à correspondre aux réalités et à être adaptés au contexte.

Il en découle un certain nombre de remarques, peut être des insuffisances, qui sont autant de sujets frappés de suspicion dans un domaine oùaborder les aspects idéologiques s'apparente, pour certains esprits pêchant par trop de scientificité, à verser dans des considérations idéologiques. N'est souvent évoqué que ce qui est légitimé comme " scientifiquement correcte ». Il s'agit ici de décrire les tendances générales et les positions de recherche les plus dominantes en matière de sociolinguistique algérienne. Ces remarques ne sont pas de facto généralisables à l'ensemble de ce qui se fait car des exceptions existent. Notre objectif ici est d'attirer l'attention sur ces points, à notre sens, pas suffisamment exploités et qui gagneraient à l'être pour atteindre à davantage de complétude dans l'appréhension des multiples facettes du plurilinguisme algérien.

Plusieurs remarques s'imposent dans ce sens, les réflexions (et non pas les considérations) d'ordre macrolinguistiques sont souvent absentes de l'analyse des faits de type micro-sociolinguistiques dans de nombreux travaux de recherche. Ces derniers pâtissent d'une absence de réflexion sur la gestion de la diglossie signalée uniquement comme un handicap pour être aussitôt éludée ce qui fait que la non-prise en charge institutionnelle de l'arabe algérien ne se pose pas, sauf pour de rares chercheurs dans le domaine. L'évolution rapide et incontrôlée de l'arabe algérien au contact du français n'interpelle pas encore, elle n'est vue que sous l'angle de la créativité et de la flexibilité plutôt que sous celui de la nonmaîtise de la langue, car ne s'agissant pas d'une langue académique/scolaire, la compétence langagière en langue maternelle ne se pose donc pas. Nous remarquerons également que le lien entre l'acquisition sereine et correcte des langues maternelles et l'apprentissage des langues étrangères n'est pas très présent dans les travaux de recherche, car il nous semble que faire le constat de ce qu'on désigne sous les appellations de « bilinguisme soustractif » (négatif) (Dabène 1994) ou de « bilinguisme additif » (positif) (Idem) devrait renvoyer non seulement à la question de l'enseignement des langues étrangères mais également aux questions de codification et d'aménagement des langues premières, or, le lien n'est pas toujours établi entre les deux questions.

L'exclusion de la problématique des langues premières du champ de préoccupation des chercheurs les amène à faire l'impasse sur la littérature véhiculée par et dans ses langues. Cette littérature continue à être qualifiée de "populaire» alors que de nombreux textes datant des cinq derniers siècles deviennent de plus en plus inaccessibles/incompréhensibles aux algérianophones d'aujourd'hui, et ce eu égard au niveau littéraire dans lequel ils avaient été élaborés et au recul de la compétence de lecture/ compréhension de ces écrits qui ne sont plus transmis en société, par les canaux traditionnels de diffusion, ni par d'autres structures pouvant prendre en charge leur transmission et leur sauvegarde. La seule littérature ayant une légitimité pour être enseignée à l'école algérienne est celle produite en arabe institutionnel ou en français, l'imaginaire maghrébin n'est accessible que par le filtre d'autres langues, s'ajoute à cela le fait que la littérature maghrébine occupe un espace modeste dans les manuels scolaires. Le dernier point que nous abordons ici concerne l'emploi de l'arabe institutionnel comme langue d'enseignement, un emploi qui n'est pas suffisamment interrogé par rapport aux langues maternelles de l'enfant arabophone et berbérophone ${ }^{11}$ pour qui cette langue est étrangère. Son emploi dès le préscolaire en milieu institutionnel n'interpelle pas, il en est de même des objectifs liés à son enseignement en vue de la fonction qu'on lui assigne dans les textes officiels ${ }^{12}$, à long terme, en société et qui est d'en faire une langue de communication dans des situations authentiques de communication.

\footnotetext{
${ }^{11}$ Il s'agit ici du domaine de recherche francophone en sciences du langage. Une enquête concernant les thématiques de recherche dans les départements d'arabe serait intéressante à initier pour voir quels sont les problèmes qui se posent à la recherche en la matière.

${ }^{12}$ Voir : http://www.joradp.dz/FTP/jo-francais/2008/F2008004.pdf
} 


\subsection{Les langues berbères :}

Pour ce qui est du domaine des langues berbères, les convergences avec le champ des études sur l'arabe institutionnel sont intéressantes à considérer, les postures liées à la saisie de la variation souffrent des mênes relents idéologiques qui figent l'état de la réflexion sur des questions urgentes. Au phénomène de polarisation, tant prégnant dans le domaine de la sphère arabophone, fait face dans certaines études, le phénomène presque contraire d'individuation linguistique ${ }^{13}$ et culturel volontaire dans la sphère berbérophone. En effet, le discours y est fondé sur une stratégie de distanciation ${ }^{14}$ par rapport aux normes linguistiques et culturelles dominantes et majoritaires en Algérie mais la satellisation est reproduite à l'intérieure de la sphère berbérophone, la variation est reconnue mais elle doit être contrôlée. Cette attitude dé déni ou de minimisation face à la variation «interdialectale » correspond aux exigences de la construction «des récits fondateurs ethniques» que Yacine Temlali définit comme «tous fondamentalement jacobins, obsédés par la recherche des éléments d'unité et l'escamotage des différences » (Temlali, 45). Notre intérêtportera particulièrement sur les processus d'individuation sociolinguistique et socioculturel qui y sont développés.

Les textes auxquels nous nous intéresserons en premier lieu sont des extraits de l'ouvrage de Salem Chaker, «Berbères aujourd'hui », réédité en 1998, un ouvrage clef dans le champ des études berbères. Il s'agit aussi d'un texte qui frappe par sa tonalité militante. Ce que nous considérons comme une relativisation du discours viendra sous la plume de Dalila Morsly et de Dourari Abderrezak. Des extraits de leurs écrits sur la question de la variation seront passés en revue.

Certaines thèses de l'aveu même de l'auteur, Salem Chaker, ne sont pas toujours fondées sur des arguments scientifiques mais obéissent à des considérations militantes voire ethnocentristes. Pour ce qui est de la variation dans les langues berbères, même s'il met en garde contre «une nouvelle situation diglossique du type de celle de l'arabe classique (arabe dialectal)»(Chaker: 166), il identifie les langues berbères comme «des dialectes » (Idem, 166) au regard des processus de fragmentation et de dialectalisation qui se seraient produits et qui sont présupposés dans l'idée de «fin $d u$ continuum linguistique berbère en Afrique du Nord » (Idem) évoquée dans le même ouvrage, il y aurait eu donc une fin du continuum berbère à un moment donné de l'histoire sauf qu'on ne peut la situer dans le temps ni encore moins prouver qu'elle avait eu lieu. L'idée est nuancée par Dourari Abderrezak :

\section{"L'Algérie historique (...) a toujours été plurilingue. Il est difficile d'imaginer un territoire aussi grand que le Maghreb (de la frontière Egypto-Libyenne jusqu'aux îles Canaries, puis au sud, le Mali, le Niger, et la Mauritanie) avec des groupes humains aussi éparpillés, qui parleraient en dépit de cela une langue unique à cette époque »(Dourari 2011).}

Non seulement l'unité supposée est problématique mais la présence d'autres langues et leurs contacts avec les langues berbères ne sont que très rarement évoqués.

La polarisation se fait à l'intérieur de la sphère berbérophone oùa variation diachronique est minimisée, réduite. «L'émergence de plusieurs standards» (Idem) régionaux serait une menace pour les dits «dialectes » (Idem) pouvant déboucher sur une/des diglossie (s) et serait une menace également pour «la promotion de la langue berbère » (Idem) ou d'une langue berbère à caractère «national » (Idem) tel que son statut est revendiqué par le mouvement berbère et dans les milieux universitaires. Pour Salem Chaker, " être Berbère aujourd'hui - et vouloir le rester- est nécessairement un acte militant, culturel, éventuellement scientifique, toujours politique ». Il défend même aux «linguistes non-Berbères» de

\footnotetext{
${ }^{13}$ «L'individuation sociolinguistique est le processus par lequel une communauté ou un groupe tend à systématiser ses différences linguistiques, à les sacraliser, à les considérer comme déterminantes, à en faire un élément de reconnaissance. Ces différences deviennent alors des indicateurs d'identité » (Marcellesi 2003 : 278). Il ajoute: "Quand la satellisation l'emporte, la variation linguistique est minimisée, hiérarchisée et subordonnée " (Idem, 281).

${ }^{14}$ Ces stratégies sont évoquées par Jean-Baptiste Marcellesi,
} 
réfléchir à la question : «Ce terrain est avant tout celui de la conscience collective des berbérophones, pour cette raison, le linguiste -surtout non Berbère- est plutôt mal venu de se substituer aux intéressés. " (Idem). L'individuation est poussée à l'extrême, tant sur le plan de l'individuation socioculturelle que sur le plan linguistique qui s’apparente à « une idéologie de la compensation » (Marcellesi 2003 : 118).

Les traits culturels distinctifs sont «une tradition orale spécifique, un patrimoine culturel, des particularités sociales... " (Chaker 1998, 16). Des éléments qu'il relativise à la fin de l'ouvrage : "tous les paramètres traditionnels qui ont permis le maintien à travers les siècles de la langue berbère (isolement géographique, organisation sociale, autarcie économique relative...) ont irrémédiablement disparu » (Idem, 205). L'auteur omet néanmoins de mentionner que les particularités socioculturelles ${ }^{15}$ caractérisent de nombreuses régions en Algérie, arabophones et berbérophones et distinguent même, parfois fortement, ces dernières entre elles, comme la composante socioculturelle targuie et kabyle, pour ne prendre que cet exemple. Pour ce qui est de la graphie, l'objectif est « d'assurer la discrimination maximale par rapport aux cultures environnantes », (Idem, 91), même si sur le plan psychopédagogique et économique, le choix n'est pas rentable, de l'aveu même du linguiste qui, d'ailleurs, avance que : «les arguments d'un théoricien semblent de peu de poids devant la volonté de matérialiser, à travers une écriture spécifique, une identité spécifique, une identité distincte », (Idem, 92).

Dalila Morsly s'intéressera à la saisie de la variation dans les études berbères, dont celles réalisées par Salem Chaker (Chaker 1991). Elle commence par appréhender le traitement de la variation à l'école à travers les objectifs assignés à l'enseignement des langues berbères. Elle constatera que la variation est reconnue mais qu'elle est amenée à être réduite à long terme : "L'unification des variantes » (Morsly 1998) devant déboucher sur "un tamazight scolaire » (Idem). La variation est donc admise mais à court terme seulement. L'argument de l'unicité historique du berbère fonctionne et opère comme un critère de légitimation de la revendication linguistique et culturelle. Cette dimension historique légitimante se heurte à la réalité évolutive et dynamique du terrain. Dalila Morsly met l'accent sur cette variation diatopique en insistant sur sa valeur symbolique en tant que constitutives «des identités régionales et même villageoises » (Idem). Elle relève le caractère passionnel lié au traitement de la variation en montrant, textes à l'appui, que les deux discours, militant et celui des décideurs, "reposent tous deux sur une conception idéaliste de la langue» (Idem). Deux arguments sont mis en avant: "La profondeur historique » et «l'argument communautaire », ce dernier convoque les attributs ethnique et linguistique liés à la berbérité.

Elle revient sur la position de Salem Chaker qui, d'après elle, « refuse d'admettre l'existence de plusieurs langues berbères et défend "une conception unitaire $d u$ berbère", en se basant sur ce qu'il nomme : «les structures fondamentales » (Chaker Salem), les pratiques linguistiques des berbérophones correspondraient à une sorte d' "abstraction linguistique et non une réalité sociolinguistique identifiable et localisable » (Morsly, 2011). Dalila Morsly conclut que «la conscience linguistique de la variation, pourtant fortement active, ne pourrait satisfaire ce désir, cette nostalgie de la langue ». (Morsly Dalila : 151). Dourari Abderrezak explique, pour sa part, que «face au puritanisme linguistique prônant l'arabe scolaire comme modèle (on a) opposé un autre puritanisme prônant "tamazight" (berbère) comme modèle unique ». Ce qui n'est pas sans nous rappeler le choix de l'arabe dès l'indépendance en remplacement du français et par opposition à lui. Ceci amène à «condamner les variétés de tamazight réellement parlées à une sorte de ghetto, celui de vulgos, alors que la gent instruite utilisera un " tamazight » différent, lui-même s'enfermant dans sa propre tour d'ivoire », une question qui commence à susciter un certain intérêt dans le domaine des sciences du langage (Lanseur 2016).

Le caractère tranché des remarques formulées ci-dessous procède d'un constat général mais il y a sx̂ement des exceptions où le traitement de la variation est relativisé, sauf qu'ils ne sont pas toujours confrontés aux positions dominantes dans le domaine de la recherche sur les langues berbères.

\footnotetext{
${ }^{15}$ Notons l'absence des autres langues de la description de la réalité du terrain linguistique et de ce qu'il qualifie d'« arabe dialectal » qui n'est évoqué qu'une seule fois, à la page 166.
} 
Les tendances que l'on peut dégager des travaux actuels sont la réification des positions de recherche sur la question des langues berbères, sur leurs statuts, ceci se traduit par l'alignement des préconisations scientifiques sur les revendications politiques liées au statut de la langue. Les avis divergents sur la question statutaire sont frappés de suspicion ou de discrédit, via le discours scientifique. Contrairement à une approche interne des structures de «la langue berbère », l'approche sociolinguistique des faits de langues, impliquant l'étude des phénomènes de contact avec d'autres langues, des représentations épilinguistiques, etc., ne conforte pas le discours unitaire sur ce qui est considéré comme l'élément le plus emblématique de l'identité. Ce recours à la linguistique interne au service d'une langue commune et la minimisation des considérations sociolinguistiques réduit le sujet parlant à un locuteur idéal, absent des préoccupations linguistiques, ceci se donne à constater à travers le développement d'une réflexion de laboratoire, théorique et théorisante, au détriment de véritables travaux de terrain. Dans le domaine de l'enseignement, la conception d'objectifs didactiques sont en déphasage avec les besoins linguistiques réels des locuteurs. L'on note dans ce sens, une convergence des objectifs didactiques liés à tamazight et les fonctions politiques qui lui sont assignés dans les textes officiels, une sorte d'alignement des objectifs d'enseignement sur les objectifs politique relevant d'une vision militante des faits de langue.

\section{Conclusion :}

Il ne sera pas ici question de conclure un débat qui, à notre sens, ne fait qu'être amorcé. Les aspects débattus par les sociolinguistes dont nous avons exposé les positions renseignent sur la nécessité de soumettre les positionnements des chercheurs à un examen critique ainsi que l'utilité de recourir à une démarche réflexive dans les travaux en sciences du langage et en didactique des langues. Ce constat procède des limites qui se sont imposées à la recherche en la matière. Ces limites se traduisent par deux faits que l'on peut constater dans beaucoup de recherches: Le premier est celui du stéréotypage des réflexions qui sont reconduites depuis des décennies sans que des éléments précis pour des politiques linguistique et éducative ne fassent consensus dans la communauté des chercheurs. Quant au second, il consiste en la prééminence parfois des considérations idéologiques dans le traitement universitaire des questions devant être appréhendées scientifiquement car posées dans un cadre académique rigoureux qui a ses règles et ses exigences. Au regard de l'aspect quantitatif croissant des recherches qui se développent dans le domaine, ainsi que l'a constaté Dalila Morsly dans ses récentes enquêtes (Morsly, 2013), il est peut-être temps d'entamer cet examen rétrospectif avant de continuer (ou tout en continuant) à faire l'analyse qualitative du réel linguistique prévalant en Algérie. A défaut d'une attitude réflexive, les contradictions résultant du recours aux deux discours, militant et scientifique, se trouvent minimisées et leurs conséquences sur la qualité de la recherche aussi. Si la complexité gagne à être assumée dans le raisonnement scientifique, le fait de revendiquer des positionnements idéologiques ne nous semble pas aider à asseoir l'utilité de ces sciences et à en préciser la pertinence en contexte algérien.

Nous clôturons ce bref exposé avec ces quelques actions et pistes de réflexions qui restent à compléter. Il nous parait important d'enseigner et de vulgariser l'Histoire des langues qui sont (ont été) pratiquées au Maghreb, sur les plans interne et externe et d'entreprendre des études comparatives sur les recherches menées dans les pays maghrébins et sur les réels socio-langagiers maghrébins. Pour une meilleure compréhension des positions de recherche, il importe d'analyser les dispositifs théoriques, notamment, qui sous-tendent la recherche dans le domaine (Morsly 2013). Comme la recherche académique peut être innervée d'idéologie, il ne serait pas vain d'attirer l'attention sur le fait que l'analyse du poids de l'idéologie dans les écrits scientifiques n'est pas une autre façon de faire de l'idéologie. Ceci passe par une déconstruction/adaptation critique du champ conceptuel de la sociolinguistique et de la didactique des langues étrangères et maternelles. Sur le plan du corpus, créer des bases de données regroupant les travaux de mémoires de master, de thèses de doctorat, des productions maghrébines et étrangères sur la question devrait permettre l'accumulation d'une matière exploitable par les chercheurs. Il s'agira également de constituer des bibliographies à partir des différentes orientations de la recherche (études macro-sociolinguistique, micro-sociolinguistique, sociodidactique, FLS, FLE, FOS, FOU..., etc.). Ces 
travaux gagneraient à être menés dans le cadre de projets de $\mathrm{PNR}^{16}$, de laboratoires, d'équipes affilées à des centres de recherche pour développer la réflexion dans le domaine et l'inscrire dans une dynamique de recherche continuelle et pluridisciplinaire.

\section{Bibliographie}

Abbes-Kara, A.Y, La variation dans le contexte algérien: Enjeux linguistique, socioculturel et didactique, in Pierozak, I., Bullot, Th., Blanchet, Ph. (Dirs.), Approches la pluralité sociolinguistique : Vers quelles convergences des pratiques de recherches et d'éducation, Rennes : Presses Universitaires de Rennes, pp. 77-86.

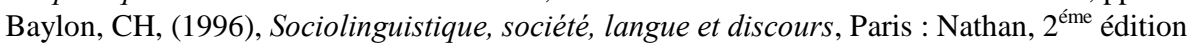

Benrabah, M, (2009), Devenir une langue dominante mondiale: un défi pour l'arabe, Genève-Paris : Librairie Droz. Benrabah, M, (1999), Langue et pouvoir en Algérie. Histoire d'un traumatisme linguistique, Paris : Séguier.

Bensekat, M, (2012), Pour une promotion du plurilinguisme en classe de langue !, in Synergie Pays du Mékong $n^{\circ} 4$, Des plurilinguismes à l'enseignement des langues. pp. 107-126.

Blanchet, $\mathrm{Ph},(2004)$, L'identification sociolinguistique des langues et des variétés linguistiques : pour une analyse complexe du de processus fatégorisation fonctionnelle, in : http://www.prefics.org/credilif/travaux/Blanchet_MIDL2004.pdf

Boch, F., \& Rinck, F., (2010), Pour une approche énonciative de l'écrit scientifique, Grenoble : Revue LIDIL de l'Université de Grenoble, 5-14.

Bourdieu, P, (2002),

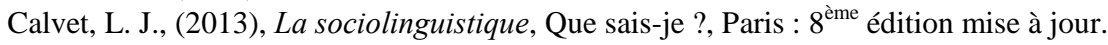

Chachou, I, (2013, La situation sociolinguistique de l'Algérie: Pratiques plurilingues et variétés à l'œuvre, Collection : Sociolinguistique, Paris : L'Harmattan.

Chachou, I, (2012), Réflexions épistémologiques autour de l'état de la dénomination et de la hiérarchisation des langues dans le discours universitaire algérien, in Socles $N^{\circ} 1$, revue du laboratoire de linguistique et de sociodidactique du plurilinguisme de l'ENS de Bouzaréah, p.p., 45-57

Chaker, S, (1998), Berbères aujourd'hui (Berbères dans le Maghreb contemporain), Paris: édition révisée, L'Harmattan.

Chaker, S, (1991), Unité et diversité de la langue berbère », Unité et diversité de Tamazight, Tome 1, Fédération nationale des Associations culturelle amazigh, pp. 129-141.

Cherrad-Benchefra, Y, (2002), Paroles d'étudiants, dans Insaniyat n 17-18, Mai-Décembre, Oran : CRASC, pp. 111-128.

Dabène, L, (1994), Repères sociolinguistiques pour l'enseignement des langues, Paris : Editions Hachette-Livre.

Dourari, A, (2016), Hétéronomie du champ du savoir ou la politique d'arabisation comme rupture avec la pensée universelle, dans Chachou I \& Stambouli M, Pour un plurilinguisme algérien intégré: Approches critiques et renouvellement épistémique, Paris : édition Rivneuve. (A paraitre).

Dourari, A, (2002), Pratiques langagières effectives et pratiques postulées en Kabylie, A la lumière des évènements $\mathrm{du}$ "printemps noir", dans Insaniyat $\mathrm{n}^{\circ}$ 17-18, Mai-Décembre, Oran : CRASC, pp. 17-35.

Elimam, A. (2004), Revaloriser les langues afin de les promouvoir, in :

http://www.barcelona2004.org/www.barcelona2004.org/esp/banco del conocimiento/docs/PO 35 FR_ELIMAM.pd f

Elimam, A, (2003), Le maghribi, alias el-daridja, langue trois fois millénaire, la langue consensuelle du Maghreb, Oran : Dar El Gharb.

Gâţă, A, (2006), Argumentation et types d'arguments dans le discours scientifique. In Adela Drăgan \& Anca Gâţă (Éds), Colloque International "Le Discours de spécialité - Théorie et pratique, Actes du Colloque (28-29 octobre 2005): 40-54. Galaţi.

Girard, A, (2013), Les manuels de langue arabe en usage en France à la fin de l'Ancien Régime, Manuels d'arabe d'hier et d'aujourd'hui. France et Maghreb, XIXe-XXIe siècle, Paris : Éditions de la Bibliothèque nationale de France, p-p, 12-26.

Messaoudi, A \& Larzul, S, (2013), Les manuels de langue arabe des débuts de l'Algérie coloniale (1830-1871), Manuels d'arabe d'hier et d'aujourd'hui. France et Maghreb, XIXe-XXIe siècle, Paris : Éditions de la Bibliothèque nationale de France, p-p, 54-78.

Kouloughli, Dj-E, (1996), Sur quelques approches de la réalité sociolinguistique arabe, in : Les langues en Egypte, $\mathrm{N}^{\circ}$ 27-28, p.p., 287-299.

Lanseur, S, (2016), Vers une nouvelle diglossie: tamazight-kabyle, in: Chachou I \& Stambouli M, Pour un plurilinguisme algérien intégré : Approches critiques et renouvellement épistémique, Paris : édition Riveneuve (A paraitre).

\footnotetext{
${ }^{16}$ Programmes nationaux de recherche.
} 
Miliani, M, (2002), Le français dans les écrits des lycéens : Langue étrangère ou sabir ? , dans Insaniyat $\mathrm{n}^{\circ}$ 17-18, Mai-Décembre, Oran : CRASC, pp. 79-95.

Morsly, D., (2012), La sociolinguistique en Algérie: Etat et perspectives, Revue Réflexions et perspectives de l'Université Alger2, pp.243-258.

Morsly, D, (2011), Enseigner la variation : L'exemple de tamazight en Algérie, Diversité n 164n CNDP- CRDP, pp. 146-151.

Morsly, D, (1997), Tamazight langue nationale?, in, Laroussi F., 1997, Plurilinguisme et identités au Maghreb, Rouen : PUR.

Morsly, D, (1983), Sociolinguistique de l'Algérie : du discours institutionnel à la réalité des pratiques linguistiques, in Calvet L-J. (éd.), Sociolinguistique du Maghreb, pp. 135-142.

Rispail, M, (2012), Interroger la sociodidactique : faux-semblants, résistances et orientations, Revue SOCLES : Reconfigurations des concepts, pour une réflexion épistémologique et méthodologique en sociolinguistique et en sociodidactique, Alger : ENS De Bouzaréah, pp.61-81.

Temlali, Y, (2015), La Genèse de la Kabylie, Aux origines de l'affirmation berbère en Algérie (1830-1962), Préface de Gilbert Meynier et postface de Malika Rahal, Edition Barazakh.

Taleb Ibrahimi, Kh. (1997) : Les Algériens et leur (s) langue (s), Alger : El Hikma.

Gérard Troupeau, (1997), Deux cents ans d'enseignement de l'arabe à l'école des langues orientales, Chroniques yéménites [En ligne], 4-5|1997, mis en ligne le 30 août 2005, consulté le 05 août 2014. URL: http://cy.revues.org/116 